\title{
Modification of the Oral Microbiota and Carcinogenesis of the Pancreas and Gastrointestinal Tract
}

\author{
Antonia Sinesi ${ }^{1 *}$, Stefania Pernarella ${ }^{2}$, Antonio Sarnataro ${ }^{3}$, Cinzia Casu ${ }^{4}$ and Savino Cefola ${ }^{5}$ \\ ${ }^{1}$ RDH Freelancer Canosa di Puglia, Italy \\ ${ }^{2} \mathrm{RDH}$ Freelancer Latina, Italy \\ ${ }^{3} \mathrm{RDH}$ and Editor Florence, Italy
}

${ }^{4}$ Private Dental Practice, Cagliari, Italy

${ }^{5}$ Private Dental Practise, Barletta, Italy

Submission: June 12, 2020; Published: June 29, 2020

*Corresponding author: Antonia Sinesi, RDH Freelancer Canosa di Puglia, Italy

Abstract

Introduction: The microbiota present in the human oral cavity can be considered one of the most complex ecosystems of our body, since the various anatomical structures present, teeth, gingival sulcus, palate, tongue and tonsils offer a variety of habitats in which different microbial agents can colonize. Some of the microorganisms present in it play a protective role for our health, offering resistance to some pathogens, helping to maintain the oral homeostasis, while others may become responsible for triggering oral diseases, like tooth decay, periodontal diseases or oral cancer. Lately the interest of the scientific world is focusing on the presence of these bacteria in tumor sites developed in distant districts, such as the gastrointestinal one. The oral microbiota and its change play an important role in the oncological pathology as for example in the carcinoma of the tongue, pharynx-esophagus, digestive tract, colorectal and pancreas.

Materials and Methods: Gastrointestinal tract cancer is one of the most common cancers worldwide. Numerous studies suggest the involvement of periodontal pathogens and cancer. In this work we considered scientific evidences and literature reviews in which the correlation between periodontal bacteria (F. nucleatum, P. ginvilalis, Tannerella forsizia, Streptococcus anginosus.) and gastro-intestinal cancer are examined and studied.

Results: Several ways in which bacteria can induce carcinogenesis have been highlighted: induction of chronic inflammation, inhibition of the immune response, interference with signaling pathways and cell cycles and support for local metabolism of carcinogens. Among the periodontal pathogenic bacteria, F. nucleatum deserves special attention. It is strongly related to gastrointestinal and pancreatic tumors. Scientific studies have shown a clear association between alteration of the oral microbiota and tumors of the gastro-intestinal tract and pancreas.

Conclusion: Bacterial biodiversity is highly associated with tumors of the gastro-intestinal tract and pancreas. Understanding this association, through the use of molecular diagnostics, can help establish a new way of preventing and diagnosing cancer based on bacterial composition. The monitoring of the microbiota could help to prevent the development of these neoplasms. Some species of the oral microbiota or alterations of the oral ecosystem could be accessible and non-invasive biomarkers for the identification of gastrointestinal tumors. The immune and inflammatory system play a crucial role in the development of the disease with a complex interaction between the host defense system and specific bacterial species. Therefore, a comprehensive understanding of the underlying mechanisms for the prevention and treatment of gastrointestinal tumors is required

\section{Introduction}

The oral cavity has more than 700 different bacterial species. These polymicrobial communities are involved in many important functions, such as immunity, protection against pathogens and the metabolism of food compounds, thus allowing homeostasis to be maintained. A close correlation between periodontal disease and systemic diseases [1] has been widely demonstrated: Cardiovascular disease, Respiratory tract infection, diabetes, rheumatoid arthritis [2], and a close correlation between oral microbiota and cancer [3]. Today cancer is the second leading cause of death in the world. The best-known bacterium associated with the development of gastric cancer in humans is Helicobacter pylori, defined as a class I carcinogen [4]. Parodontal bacteria such as Prevotella sp., Tannerella forsythia, Porphyromonas gingivalis Fusobacterium nucleatum and Porphyromonas gingivalis 
are strongly associated to the development of intestinal and pancreatic cancer [5].

\section{Materials and Methods}

The oral cavity and the entire digestive system, even if represented by different anatomical regions, are highly colonized by several microbes. However, studies indicate that oral bacteria are able to spread throughout the digestive tract to reach the colon, the last stretch of this apparatus. It is interesting to note that periodontal bacteria $P$. gingivalis and Aggregatibacter actinomycetemcomitans have been linked to the development of digestive tumors such as primary pancreatic adenocarcinoma [6]. Instead species such as Fusobacterium nucleatum have large invasive properties for intestinal mucosa and involvement in the development of carcinogenesis [6,7]. Brandilyn A. Peters in 2017 in a prospective study nested in two cohorts showed that Porphyromonas gingivalis is strongly associated with an increased risk of esophageal squamous cell carcinoma (ESCC) while Tannerella forsythia is associated with a higher risk of esophageal adenocarcinoma [8] (EAC).

Fan X et al. [9] selected 361 pancreatic adenocarcinoma incident and 371 controls matched by two prospective studies for Porphyromonas gingivalis and Aggregatibacter actinomycetemcomitans, were associated with higher risk of pancreatic cancer [9]. Recent epidemiological studies have shown that poor oral health status and specific blood antibodies for oral pathogens are associated with an increased risk of pancreatic cancer [10]. Many scientific evidences show that history of periodontal disease and tooth loss are associated with an increased risk of pancreatic cancer [11]. Providing further evidence of an association of pancreatic cancer risk with $P$. gingivalis, a large european cohort found that an elevated concentration of $P$. gingivalis serum antibodies were associated with a double increase in cancer risk to pancreas [12]. F. nucleatum expresses Fad A, an adhesion component of the bacterial cell surface that binds the cadherin $\mathrm{E}$ host, leading to the activation of Z-catenin [13]. F. nucleatum (Fn) binds E-cadherin and promotes CRC cell proliferation [14].

\section{Results}

In the colon, periodontal bacteria can alter the composition of the microbiota, causing an initial intestinal dysbiosis, which triggers aberrant immune and anti-inflammatory responses, which over time can be the trigger or cause of the development of a tumorigenesis process charged to these districts [15]. Swidsinski [16] reported, using fluorescence in situ hybridization, that a dense multispecies bacterial biofilm, including oral bacterial types, was present inside the pancreatic duct of patients with calcific pancreatitis; however, the reason why the presence of bacteria of the same clonal origin, found in the pancreas and in the oral cavity, is unknown [16]. These bacterial types are found in the atherosclerotic plaques $[17,18]$ distal esophageal tissue [19], the brains of Alzheimer's patients [20], and in the unit fetal-placental. From the studies examined it seems that the microbes present in the mucosal sites can become part of the tumor microenvironment of the neoplasm of the aerodigestive tract, and intratumoral microbes can influence the growth of the cancer and its spread [13]. The surfaces of the digestive tract are subjected to continuous abuse, infections from lesions, and this can destroy the integrity of the mucous membranes themselves. Under normal physiological conditions, barrier lesions represented by mucous membranes are rapidly repaired and tissue homeostasis is restored. In cases where this repair does not take place, the imbalance between the bacterial species contributes to the continuous lesion of the barrier and the failure to restore homeostasis and it is precisely in these contexts that the microbiota can influence carcinogenesis [13].

Three different ways by which microbiota contribute to carcinogenesis have been identified, increasing, or decreasing a host's risk:

1. Altering the balance between proliferation and death of host cells.

2. Disturb the functionality of the immune system.

3. Affecting the metabolism within the host [13].

\section{Conclusion}

Deepening this association between periodontal bacteria and cancer of the gastro-intestinal tract, through the use of molecular diagnostics can help establish a new concept for the prediction or diagnosis of cancer based on bacterial composition. The microbiological research consists in quantitatively and qualitatively detecting, in the sample taken, the concentration of specific periodontal pathogenic bacteria, using the PCR technique in real time (16S rRNA) and / or the search for specific highly sensitive antibodies of periodontal pathogenic bacteria. Therefore, some species of oral microbiota or displacement of the oral ecosystem can also serve as easily accessible and non-invasive biomarkers for highlight high risk of gastrointestinal tumors. The oral cavity therefore plays a fundamental role because it is involved in correlations with systemic diseases and tumors. Cancer prevention requires a multidisciplinary approach that includes various health professionals such as the doctor and nutritionist, but an evaluation of the oral cavity by the dental hygienist and dentist could play a key role in the prevention of gastrointestinal cancer and pancreas.

\section{References}

1. Carter CJ, France J, Crean S, Singhrao S (2017) The Porphyromonas gingivalis/Host Interactome Shows Enrichment in GWASdb Genes Related to Alzheimer's Disease, Diabetes and Cardiovascular Diseases. Front Aging Neurosci 9: 408.

2. Seesandra V, Rajagopala, Sanjay Vashee, Lauren M, Oldfield, et al. (2017) The Human Microbiome and Cancer. Cancer Prev Res (Phila) 10(4): 226-234 
3. Gómez-Bañuelos E, Mukherjee A, Darrah E, Andrade F (2019) Rheuma-toid Arthritis-Associat-ed Mechanisms of Porphyromonas gingivalis and Aggregatibacter actinomycetemcomitans. J Clin Med 8(9): 1309.

4. Burucoa C, Axon A (2017) Epidemiology of Helicobacter pylori infection. Helicobacter.

5. Fan X, Alekseyenko AV, Wu J, Peters BA, Jacobs EJ, et al. (2018) Human oral microbiome and prospective risk for pancreatic cancer: a population-based nested case-control study, Gut 67(1).

6. Burkhardt Flemer, Ryan D Warren, Maurice P Barrett, Katryna Cisek, Anubhav Das, et al. (2018) The oral microbiota in colorectal cancer is distinctive and predictive. Gut 67(8): 1454-1463.

7. Komiya Y, Shimomura Y, Higurashi T, Sugi Y, Arimoto J, et al. (2019) Patients with colorectal cancer have identical strains of Fusobacterium nucleatum in their colorectal cancer and oral cavity. Gut 68(7): 13351337.

8. Peters BA, Wu J, Pei Z, Yang L, Purdue MP, et al. (2017) Oral Microbiome Composition Reflects Prospective Risk for Esophageal Cancers Cancer Res 77(23): 6777-6787.

9. Castellarin M, Warren RL, Freeman JD, Dreolini L, Krzywinski M, et al. (2012) Fusobacterium nucleatum infection is prevalent in human colorectal carcinoma. Genome Res 22(2): 229-306.

10. Zhang Y, Wang X, Li H, Ni C, Du Z, et al. (2018) Human oral microbiota and its modulation for oral health. Biomed Pharmacother 99: 883-893.

11. Michaud DS, Izard J, Wilhelm-Benartzi CS, You DH, Grote VA, et al. (2013) Plasma an-tibodies to oral bacteria and risk of pancreatic cancer in a large European prospective cohort study. Gut 62(12): 17641770 .
12. Garrett WS (2015) Cancer and the microbiota. Science 348(6230): 8086.

13. Rubinstein MR, Wang X, Liu W, Hao Y, Cai G, et al. (2013) Fusobacterium nucleatum promotes colorectal carcinogenesis by modulating E-cadherin/b-catenin signaling via its FadA adhesin. Cell Host Microbe 14(2): 195-206.

14. Swidsinski A, Schlien P, Pernthaler A (2005) Bacterial biofilm within diseased pancreatic and biliary tracts. Gut.

15. Koren O, Spor A, Felin J (2011) Human oral, gut, and plaque microbiota in patients with atherosclerosis. Proc Natl Acad Sci USA.

16. Marcelino SL, Feitosa AC (2009) Quantitative detection of periodontopathic bacteria in atherosclerotic plaques from coronary arteries. J Med Microbiol 58(Pt 12): 1568-1575.

17. Pei Z, Bini EJ, Yang L (2004) Bacterial biota in the human distal esopha-gus. Proc Natl Acad Sci USA 101(12): 4250-4255.

18. Riviere GR, Riviere KH, Smith KS (2002) Molecular and immunological evidence of oral Treponema in the human brain and their association with Alzheimer's disease. Oral Microbiol Immunol 17(2): 113-118.

19. Aagaard K, Ma J, Antony KM (2014) The placenta harbors a unique micro-biome. Sci Transl Med 21(6): 237.

20. Russo E, Bacci G, Chiellini C, Fagorzi C, Niccolai E, et al. (2018) Preliminary Comparison of Oral and Intestinal Human Microbi-ota in Patients with Colorectal Cancer: A Pilot Study Front Microbiol 8: 2699. 